\title{
A Design of the Traffic Lights Intelligent Control System Based on ARM and Zig Bee
}

\author{
Fensu Shi \\ Beifang University of Nationalities \\ Yinchuan, China \\ E-mail:shifensu@163.com
}

\author{
Ningning Ge \\ Beifang University of Nationalities \\ Yinchuan, China \\ E-mail:gening19890715@163.com
}

\begin{abstract}
With the rapid development of economy,Road traffic environment is changing slowly, the efficiency of the people and car traffic is becoming more and more aroused people's attention,As one of important infrastructure of road network,traffic lights have a great impact on traffic efficiency.In the traditional traffic lights control system,the control of people and Car traffic was through relatively reasonable fixed lights switch time, after the traffic statistics, analysis of the history of road intersections. This can lead to reduce the traffic efficiency,such as, there is a large flow in the red light direction but a small flow in the green light direction at the same time,Besides, The traditional control system adopts embedded wire method on the system arrangement mostly, This means the high cost in maintenance and maintenance.On the basis of retain fixed switching time, This design scheme based on ARM, Zig-bee wireless network technology and magnetic sensors to improve the traffic efficiency through the real-time collection, analysis on the road traffic,according to the results of real-time adjust the traffic lights control strategy.
\end{abstract}

Keywords- component: Zig-bee; intelligent control; magnetic induction; intelligent transportation

\section{INTRODUCTION}

In recent years, more and more cars into the crowded city road, although, ITS (Intelligent Transport System) ${ }^{[1]}$ is a kind of advanced integration of integrated transportation management System. It make the road to optimal use by means of sensing, information, electronic etc. However, with the reality of Chinese condition, only a fraction of few coastal cities have the strength for the corresponding research, it is very rare to applicate ITS to the real life. More and more crowed cities improve the efficiency of road traffic is urgent, It has deeply influenced People's Daily life. So, In this paper, I desigen an intelligent traffic light control system based on ARM and Zig-bee. it can improve the efficiency of traffic, ease the contradiction among people, vehicles and road.

\section{GENERAL DESIGN OF SYSTEM}

This system mainly consists of the following sections:

a)ARM control unit+Zig-bee wireless communication unit+Lights control unit

b)Traffic acquisition unit+Zig-bee wireless communication unit

c)Related software

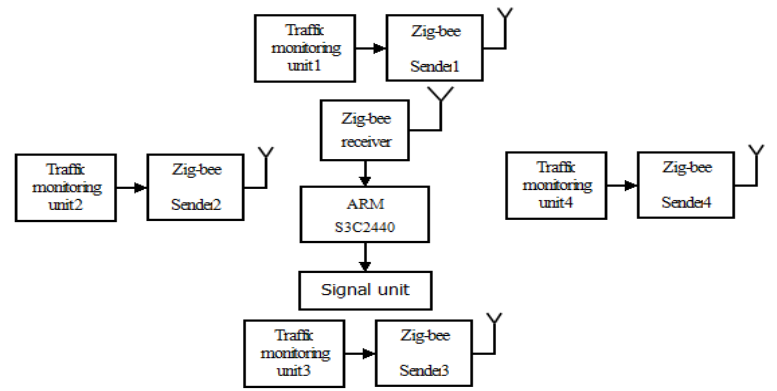

Figure 1. system structure

\section{THE DESIGN OF CONTROL UNIT}

ARM main control board uses the samsung's S3C2440 chip, the S3C2440 is developed with ARM920T core, a 32-bit RISC processor designed by Advanced RISC Machines ${ }^{[2]}$,CPU frequency up to $533 \mathrm{MHz}$.It integrates three serial ports, SD card controller, the USB Host controller, Lcd controller, NandFlash controller and so on. Besides, It also includes industrial control bus, Camera controller, PCMCIA interface, can be connected by other bus equipment. It meets the system requirements in theory. The control unit receive traffic information from traffic detection unit in all directions through the Zig-bee wireless communication units, then determine the corresponding signal control strategy at the next light switch cycle after classifying and summary, so as to realize intelligent control. The specific design below:

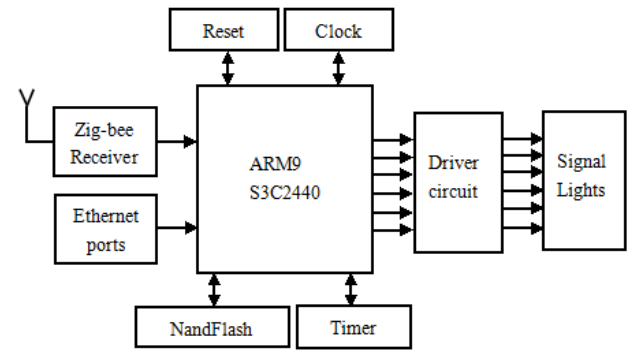

Figure 2. control unit structure

There are two aspects to the main purpose of the master control unit:

First, receive real-time traffic data analyze traffic then formulate the corresponding control strategy;

Second, store the data and control the light according to the latest control strategy. 
The design adopts $74 \mathrm{HC} 573$ drive chip to drive signal chy-tech, it contains eight 3-states of inversion of transparent latch, is a kind of high performance silicon devices. Latch is transparent to the data when LE is high, the output is the input. On the contrary, Output control is not affected by latch internal operation, namely the old data to maintain at the same time, new data can be input. This is qutite fit to the requirement of the system. Drive circuit diagram as shown in the figure below:

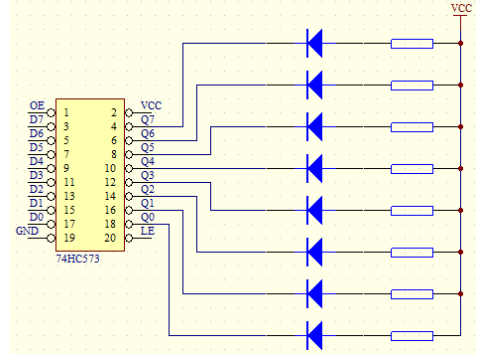

Figure 3. lights drive circuit diagram

\section{ZIG-BEE WIRELESS COMMUNICATION TECHNOLOGY}

Zig-bee is a kind of highly reliable wireless data transmission network ${ }^{[3]}$, communication distance from a standard 75 meters to hundreds of meters, a few kilometres, and support unlimited extension. It has the characteristics of low power consumption, easy networking. This solution adopts CC2530 chip in Zig-bee module which provides driver of the MAC and PHY layer, communicate with the main controller by the way of SPI.

Main features following:

a)Powerful DMA channel 5

b)IEEE802.15.4 MAC timer, three general timer

c)Which has the function of capture $32-\mathrm{MHz}$ sleep timer

d)Hardware support for CSMA/CA

e)Supports accurate digital received signal strength indicator (RSSI)/link quality indicator (LQI)

f)8-road input and configurable 12 bit ADC

g)high sensitivity, low power, etc

Zig-bee communication network in this system mainly includes the coordinator node and sensor node. The coordinator node comply with IEEE802.15.4 agreement, it is responsible for establishing the wireless network, maintenance of all sensor nodes, accept data from each sensor node and the data to the main control unit. Sensor nodes send traffic to the coordinator after $\mathrm{A} / \mathrm{D}$ conversion. Wireless network structure is as follows:

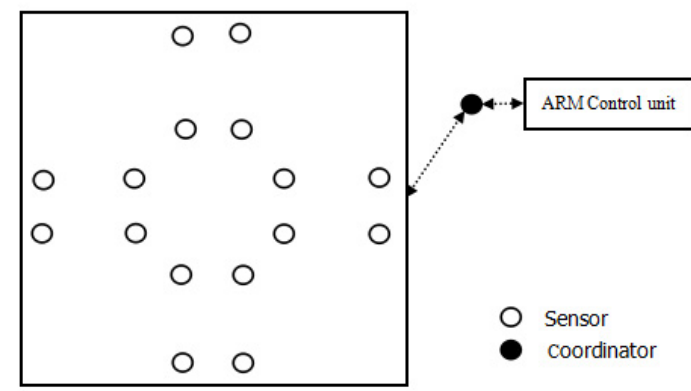

Figure 4. wireless network structure diagram

\section{THE DESIGN OF TRAFFIC DETECTION UNIT}

The system flow detection is mainly used magnetic sensors, this is a new type of vehicle detection sensors ${ }^{[4]}$.It to determine whether a vehicle through by testing vehicles on geomagnetic signal to the perturbation, compared to other sensors, it is easy to install, maintain, and little damage to the road. Besides, Monitor can be installed hanger or on the roadside, this is in ensuring accuracy at the same time has good anti-interference.

\section{MAGNETIC SENSOR}

Magnetic sensor is a kind of device, which points out that the information such as posture and movement direction of the object to be tested by sensing the magnetic field distribution of change.

There are mainly several kinds of technology to detect the magnetic field distribution change principle, Magnetic resistance effect, Hall effect, Electromagnetic induction, AMR(alien magnetic reaction) ${ }^{[5]}, \mathrm{GMR}$ (giant magnetic reaction)etc. This scheme uses HMC1002 based on AMR sensor ${ }^{[6]}$.It can quickly detect the static magnetic field, access to the direction of the magnetic field, size etc at the same time. It has small volume, low price and high sensitivity $(10 \mathrm{mv} / \mathrm{v} / \mathrm{mT})$, linear error in $0.05 \% \sim 1.6 \%$.In theory, this fully meet the system requirements. The specific design the diagram below:

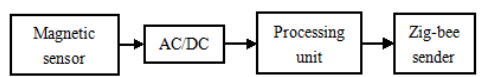

Figure 5. traffic detection module structure

Flow detection methods ${ }^{[7]}$ :Laid two magnetic sensors in each lane.A near stop mark, another is located in the driveway, 200 meters distance from intersection. When the vehicle through the sensor 1 , the lane traffic count plus one, when vehicles through sensor 2 , also add a traffic count. So, the difference of the two is the current flow of the lanes. 


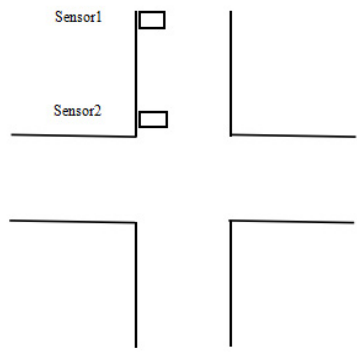

Figure 6. sensors' layout diagram

\section{THE DESIGN OF SOFTWARE RELATED TO SYSTEM}

The ARM board is equipped with $\mu$ CLinux an embedded operating system, this is a downsizing of a common version Linux. The kernel code is more compact. It retain the advantages of many common Linux, such as network functions, portability, system stability, and so on. In the control system, the control strategy of dynamic implementation is the key, also the difficulty. The algorithm is realized in general Linux, then ported to $\mu$ CLinux OS after cross compiling. The following for the realization of the algorithm.

The overall train of thought: According to the fixed switching time to ensure the normal traffic order when crossing traffic smaller. Extend green time when the vehicle is large on the direction to improve traffic efficiency and reduce energy consumption. In one direction, for example: According to the fixed switch time 30 s run normally without waiting for the green light by vehicles. Judge waiting for traffic $\mathrm{S}$ during the red light,

If $S<=8$, then adjust the green light time of $40 \mathrm{~s}$ in the next cycle

If $8<\mathrm{S}<=13$, then adjust the green light time of $55 \mathrm{~s}$ in the next cycle

If $13<\mathrm{S}<=18$, then adjust the green light time of 70 s in the next cycle

If $\mathrm{S}>18$, then adjust the green light time of $90 \mathrm{~s}$ in the next cycle.

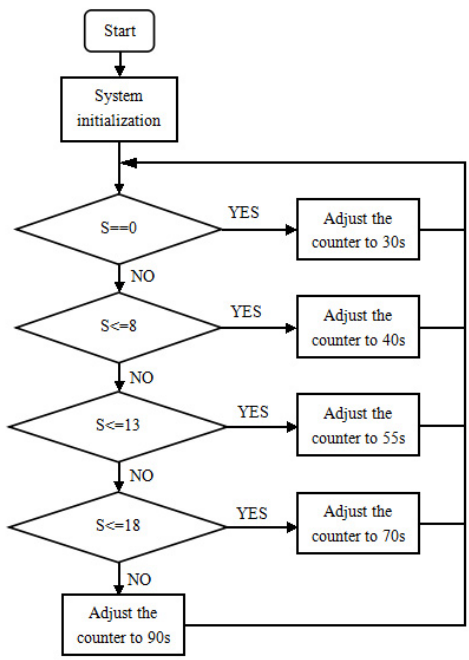

Figure 7. the flow chart of figure strategy adjustment

\section{CONCLUSION}

This plan has realized the optimization of a single intersection traffic, does not implement the overall optimization, but keep the interface to the whole optimization. The control module can upload traffic information to traffic command center through the Ethernet interface, GPRS or Zig-bee. Adjust the light of the optimized control strategies in the command center in order to realize the global optimization of city road network. In this paper, on the basis of the combined with actual situation in our country, we Put forward a kind of intelligent traffic light control system scheme based on the Zig-bee wireless network transmission technology and magnetic sensors. There is a certain significance in relieve the contradictions among people, vehicles, roads, improvment of the traffic efficiency, improvment of the intelligent tarffic under the condition of the traffic enviroment increasingly complex, ITS application is not fully mature. This design has good practical value because of the technology have been fully involved in research.

\section{ACKNOWLEDGEMENT}

It is a project supported by National Natural Science Foundation pre-breeding program(Grant No.2012QZP01) and School-enterprise cooperation in scientific and technological breeding project(Grant No.2012XQG2) of Beifang University of Nationalities.

\section{REFERENCE}

[1] Li Shuguang.Liu Yuncai.On the Development of Intelligent Transportation[J].2005.21(6)

[2] Hou Dongqing.The Design of Cross road Traffic Signal Lights Based ARM9[J].2007.20(2)

[3] Zhang Jian.Application in Intelligent Signal Lamp Control Based on the ZigBee Wireless Network Technology[J].2013.2

[4] Ma Shanshan.Cheng Mingxiao.Bao Li.Research on Vehicle detection system based on Geomagnetic sensor. Machine and hydraulic pressure $[\mathrm{J}] .2012 .40(2)$

[5] Zhang Xiaosen.Research and implementation of vehicle detection algorithm based on magnetic resistance sensor.Beijing:Beijing jiaotong university[D].2012.7.18-22

[6] Dong Yu.The Research and Application of Dual-axis Magnetoresistive Sensor Based on HMC1022.Jilin:Jilin university[D].2009.5.12-20

[7] Wen Zhida.Liang Guirong.Chen Biming.Gao Suping.The Traffic Signal Control Based on the Flow Rate of Vehicles.Automation Technology Institute[J].2009.28(6) 